\title{
RECENT AND FUtURE X-RAY OBSERVATIONS \\ of Classical Novae During the OUtburst Stage
}

\author{
Hakkı Ögelman \\ Max-Planck Institut für extraterrestrische Physik \\ D-8040, Garching, F.R.G.
}

The discovery of soft X-rays from Nova GQ Mus with the EXOSAT satellite has added an important channnel to the observational information on classical novae during the outburst stage (Ögelman, Beuermann, and Krautter 1984). With peak luminosities in excess of $10^{38} \mathrm{erg} \mathrm{s}^{-1}$ and total liberated energies of more than $10^{45} \mathrm{erg}$, novae constitute the second most energetic outburst phenomena in the galactic population exceeded only by supernovae (see Gallagher and Starrfield, 1978; Truran, 1982 for a review of the observations and theory). Regardless of the details of the outburst, objects of the size of white dwarfs $\left(\sim 10^{9} \mathrm{~cm}\right)$ radiating close to Eddington luminosities $\left(\sim 10^{38} \mathrm{erg} \mathrm{s}^{-1}\right)$ should emit soft X-rays provided that the envelope is sufficiently transparent. In addition, the ejected shell, moving at velocities around $1000 \mathrm{~km} \mathrm{~s}^{-1}$, is capable of shocking the circumstellar medium to X-ray temperatures.

Despite these expectations, hard X-ray observations of classical novae during outburst, have yielded only upper limits, indicating that these objects were not copious emitters in the 2 to $6 \mathrm{keV}$ range (Hoffman et al. 1976; Cruise 1977). In the soft X-ray region (0.1-4.0 keV), sufficient sensitivity has only been available after the advent of space-borne focusing telescopes such as EINSTEIN and EXOSAT. Since most of the currently discovered novae are at distances of few kiloparsecs and in the galactic plane, absorbtion by the cold interstellar gas, further complicates the soft X-ray detection problem.

The only classical nova observed by the EINSTEIN satellite close to the outburst was V1500 Cyg. In spite of an earlier claim of non-detection (Hutchings 1970), Chlebowski and Kałużny (1988) have re-analyzed the EINSTEIN IPC data and shown that V1500 Cyg was seen at a $3 \sigma$ level of $0.011 \pm 0.004 \mathrm{cts} \mathrm{s}^{-1}, 1390$ days after its optical maximum. The first discovery of soft X-rays from classical novae during the outburst stage was accomplished with the EXOSAT satellite where Nova GQ Mus was detected at about 460 days after outburst (Ögelman, Beuermann and Krautter 1984). With further observations of the three brightest novae whose outburst stage coincided with the lifetime of the EXOSAT satellite, namely Nova GQ Mus, Nova PW Vul and Nova QU Vul the soft X-ray light curve of classical novae were sampled from optical maximum to $\sim 900$ days after (Ögelman, Krautter and Beuermann 1987). Figure 1 shows the thin Lexan counting rates for the three novae as a function of elapsed time since optical maximum.

Two general conclusions about the time dependence of soft $\mathrm{X}$-rays can be drawn from Figure 1: i) for Nova GQ Mus we start observing $\sim 460$ days after the outburst. In the interval $\sim 460$ to 


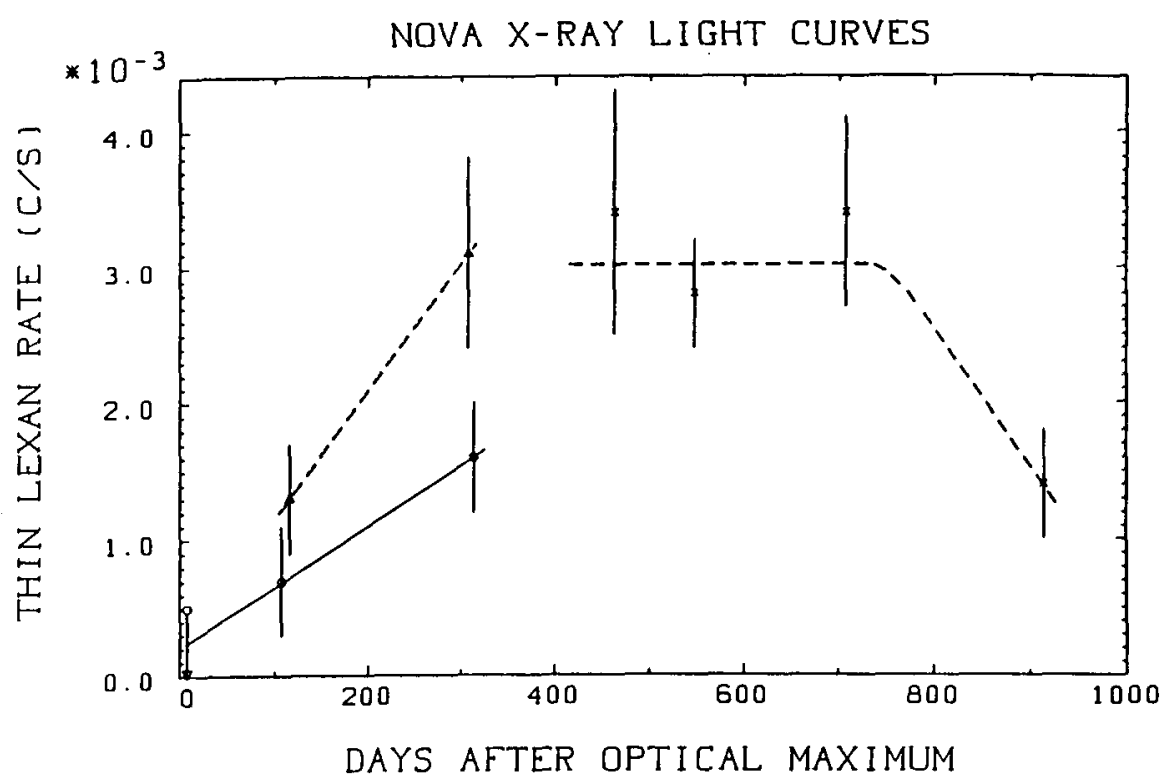

Figure 1: Counting rates of the EXOSAT low-energy telescope with the thin Lexan filter as a function of elapsed time since optical maximum for the three novae: $(x)$ Nova GQ Mus ; (०) Nova $P W V u l ;(\triangle)$ Nova $Q U$ Vul (from Ögelman, Krautter and Beuermann 1987)

$\sim 700$ days, the emission is relatively constant and subsequently starts to decay with a timescale $\sim 300$ days. ii) for Nova PW Vul and Nova QU Vul observations from outburst to $\sim 300$ days show the emission increasing linearly with time.

If we assume that Nova GQ Mus shows a typical nova X-ray light curve, and it had a similar risetime behaviour to Nova PW Vul and Nova QU Vul during the first $\sim 400$ days after outburst, Figure 1 implies that the X-ray emission from novae increases from $\sim 0$ at optical maximum to a plateau, with a timescale of 300 to 500 days, remains constant for about 300 to 500 days, then decays with a timescale of $\sim 300$ days. The fact that only the last data point shows a $\sim 3 \sigma$ reduction in the counting rate is not a strong evidence for a permanent decrease in the X-ray flux. Since we cannot foresee any other obvious effect that may cause a temporary decrease, and since we know that novae do eventually turn-off, to a first approximation, we assume that this decrease represents a permanent turn-off of the X-ray flux. These observations seem best explained by the constant bolometric luminosity model of a hot white dwarf remnant. Although these measurements suffered from limited statistics, very broad energy bandpass, and incomplete sampling of any single nova, their constraints on the theories of nova outburst were significant (see Starrfield, 1988 for a review and further references). One constraint suggested by the decrease of Nova GQ Mus flux 900 days after the optical maximum is that the lifetime of the white dwarf remnant is $\sim 2$ to 3 years, which leads to the conclusion that the burned envelope mass $M_{b u r n}$ should be of the order of $10^{-6} M_{\odot}$. There is however, some indirect evidence from recent optical spectra of Nova. GQ Mus suggesting that the central source has continued to increase in temperature (Krautter and Williams 1989), thus contradicting the turnoff interpretation of the X-ray data. The second 
constraint of X-ray data is that the maximum temperature of the white dwarf remnant should approximately be within $200000 \mathrm{~K}$ to $400000 \mathrm{~K}$. A white dwarf remnant evolving like the central star of a planetary nebula, with core mass of 0.8 to $0.9 M_{\odot}$, core luminosity of $\sim 2 \times 10^{4} L_{\odot}$, and envelope mass of $10^{-6} M_{\odot}$, can explain the general characteristics of the X-ray measurements. In order to have larger white dwarf core masses, estimated from the thermonuclear runaway models, a wind with $\dot{M} \leq 5 \times 10^{-7} M_{\odot} \mathrm{yr}^{-1}$ appears to be necessary so as to keep the temperatures down in the observed range.

In addition to the classical novae, EXOSAT has detected RS Oph, a recurrent nova up to 250 days after outburst (Mason et al. 1986). In contrast to the low count rates from the classical novae, RS Oph showed initially a strong flux which declined in $\sim 150$ days to the low level of the classical novae. The initial maximum of the X-ray flux has been interpreted as thermal bremsstrahlung from the shock heated circumstellar gas from previous explosions, while the residual X-ray emission after $\mathbf{2 5 0}$ days has been interpreted as coming from the surface of a hot white dwarf remnant.

One further point in the $\mathrm{X}$-ray light curve of classical novae comes from the recent work of Chlebowski and Katuzny (1988); an EINSTEIN detection for V1500 Cyg, 1390 days after outburst. The authors have interpreted the countrate of $0.011 \mathrm{cts} \mathrm{s}^{-1}$ as due to a thermal bremsstrahlung spectra with $k T \sim 1-10 \mathrm{keV}$ and concluded that it corresponds to a luminosity of $1.2 \times 10^{32}$ erg s${ }^{-1}$ at the assumed distance of $1.2 \mathrm{kpc}$ and $N_{H}=2.9 \times 10^{21} \mathrm{~cm}^{-2}$. To check the hot white dwarf model, I have folded the blackbody fit parameters of the EXOSAT data for Nova GQ Mus plateau with the EINSTEIN response. Indeed, for a white dwarf with radius $\sim 10^{9} \mathrm{~cm}$ and an effective temperature of $\sim 300000 \mathrm{~K}$ I get similar countrates to that observed by EINSTEIN . The implied luminosity is $6 \times 10^{36} \mathrm{erg} \mathrm{s}^{-1}$. Thus, the X-rays from Nova V1500 Cyg could still be interpreted as due to a cooling or nuclear burning white dwarf about 4 years after outburst.

The alternate possibility of explaining the X-ray emission may be the shocked circumstellar material model. This model was initially proposed by Brecher, Ingham, and Morrison (1977) in order to explain the transient soft X-ray sources where it is predicted that as the ejected nova shell moves through the circumstellar gas, it will heat it to characteristic temperatures around a few $\mathrm{keV}$ and generate thermal bremsstrahlung radiation. If the X-rays detected by EXOSAT are due to bremsstrahlung radiation with $\mathrm{kT} \sim 1$ to $3 \mathrm{keV}$ the luminosities 300 to 500 days after the outburst are $4 \times 10^{34}, 4 \times 10^{33}$, and $10^{34} \mathrm{erg} \mathrm{s}^{-1}$ for Nova GQ Mus, Nova PW Vul, and Nova QU Vul respectively. with corresponding emission measures in the $10^{56}$ to $4 \times 10^{57} \mathrm{~cm}^{-3}$ range. With expansion velocities of 800 to $1100 \mathrm{~km} \mathrm{~s}^{-1}$, the spherical volume shocked after 300 to 500 days is about $4 \times 10^{46}$ to $4 \times 10^{47} \mathrm{~cm}^{3}$. The electron densities required to give such emission measures are 0.5 to $1 \times 10^{5}$ electrons $\mathrm{cm}^{-3}$; the cooling time for a plasma with this density is about 30 to 60 years. This cooling timescale appears to conflict with the decline of the Nova GQ Mus flux after $\sim 2$ years, and the EINSTEIN luminosity of $1.2 \times 10^{32} \mathrm{erg} \mathrm{s}^{-1}$ (for thermal bremsstrahlung spectrum) for Nova V1500 Cyg, measured about 4 years after the optical maximum. In case the shocked gas is in denser filaments, such as in the supernovae shells, the time scale of emission may be reduced by the square root of the volume filling factor. However, the gas has to be clumpy with a filling factor of 100 in order to bring the estimated cooling timescale down by a factor of 10 . 
Despite the attractive features of the hot white dwarf remnant model, the slow rise and decay timescales of the X-ray light curve are puzzling. I have attempted to simulate the X-ray light curves with a simple model. I take a hydrogen burning white dwarf with $1 M_{\odot}$ core mass and luminosity $\sim 10^{38} \mathrm{erg} \mathrm{s}^{-1}$ (Paczyński 1971). On top, I add various starting envelope masses ranging from $10^{-7}$ to $10^{-4} M_{\odot}$ in hydrostatic equilibrium. I estimate the envelope structure and hence the effective temperature using the $n=3$ polytrope model of McDonald (1980). Subsequently I allow the envelope mass to decrease according to the hydrogen burning rate and calculate the X-ray light curve that EXOSAT would see. After the envelope is exhausted, I allow the white dwarf to cool with a timescale of $\sim 10 \mathrm{y}$. Regardless of the initial conditions, the striking feature of the $\mathrm{X}$-ray light curves is that once they appear, around the time when the envelope mass has decreased to $10^{-6} M_{\odot}$, they rise very fast to the maximum and then decay with a similar timescale. The reason for this behavior is basically due to the fact that the $\mathrm{X}$-ray detectors are sampling the Wien end of the spectrum where the flux is exponentially dependent on the effective temperature as $e^{-\frac{E}{k T}}$. Thus, as the white dwarf remnant becomes hotter during its progress along the constant luminosity track, it moves into the X-ray window with exponential intensity increase; subsequently it moves out again with an exponential decrease as it cools at constant radius. Starting with larger envelope masses, one can delay the arrival time of the X-ray spike or by adding additional wind losses from the envelope one can bring it to earlier times, however the characteristic shape of the exponential rise and decay times and the duration remains the same. Figure 2. shows two such $\mathrm{X}$-ray light curves.
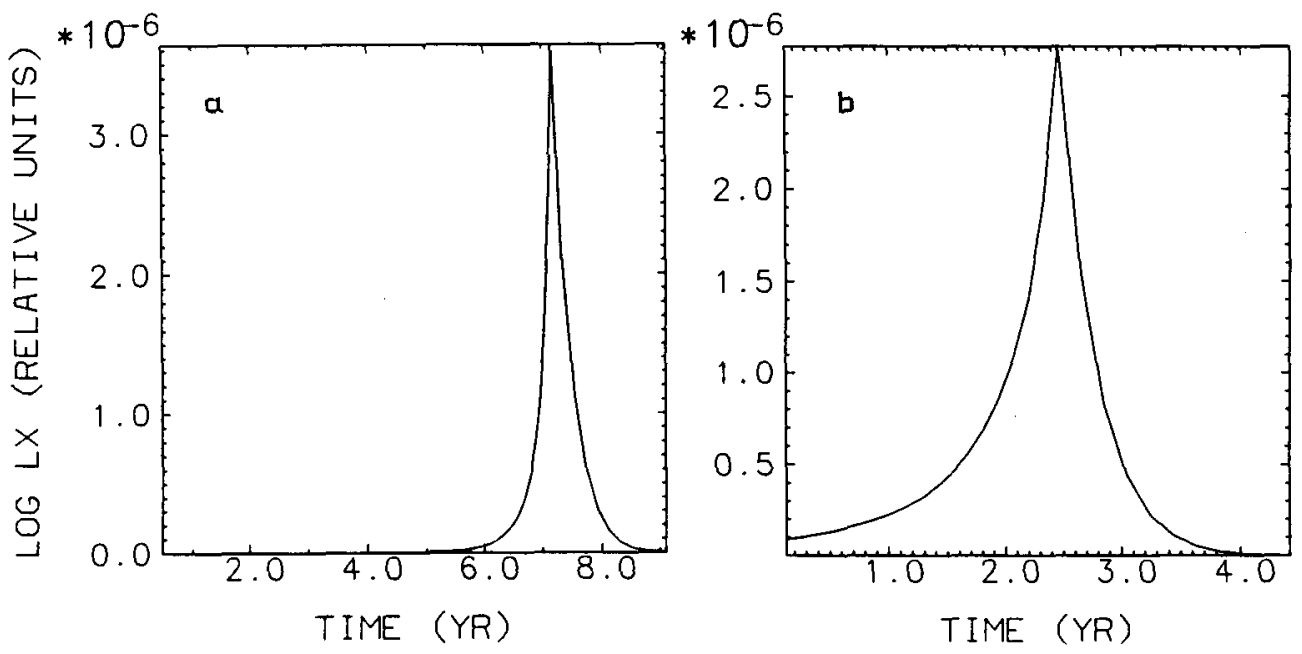

Figure 2: Two simulated $X$-ray light curves for the constant bolometric luminosity model for $1 M_{\odot}$ cores: a) initial envelope mass of $10^{-5} M_{\odot}$ and a wind of $2 \times 10^{-6} M_{\odot} y^{-1}$; b) initial envelope mass of $10^{-6} M_{\odot}$ and no wind. The cooling timescale in both cases was assumed to be $10 \mathrm{y}$.

These predicted curves differ significantly from the slow rise and decay times observed (see Figure 1). Although the model is simple and naive, the arguments that determine the shape of the $X$-ray light curve are fundamantal. 
We have initially disfavored the shocked circumstellar gas model for X-ray emission mainly because it predicts a longer timescale than that observed. On the other hand, the hot white dwarf remnant model does not produce the observed X-ray light curves either. Does reality consists of a mixture of these two emission processes; are there other processes that we have not considered; or do we have to just make more refined models for the two processes considered? It is clear that more experimental and theoretical work are necessary to resolve these issues.

On the experimental side the existing measurements suffer from lack of spectral information: EINSTEIN observed only one nova at a marginal level while EXOSAT had insufficient energy resolution and poor photon statistics. I want to conclude this contribution with a short discussion of the ROSAT satellite mission (Trümper 1983), a focusing soft X-ray telescope that is capable of adding very significant information to our knowledge of the $\mathrm{X}$-ray emission of classical novae during outburst. Scheduled for launch around March 1990, the ROSAT payload will consist of an $\mathrm{X}$-ray telescope (XRT) with a geometric area of $1141 \mathrm{~cm}^{2}$ sensitive to photons in the 0.1 to $2 \mathrm{keV}$ and an extreme UV telescope (WFC) with $456 \mathrm{~cm}^{2}$ geometric area, sensitive to photons in the 0.02 to $0.4 \mathrm{keV}$ range. Since most of the currently discovered novae are at distances of few kiloparsecs and in the galactic plane, absorbtion by the cold interstellar gas will most likely not allow detection of novae with the WFC. The XRT on the other hand, should be an excellent instrument to detect classical novae during outburst stages. Furthermore, the ROSAT XRT with the Position Sensitive Proportional Counter (PSPC) at the focus should be able to differentiate easily a soft blackbody spectrum from a hard thermal bremsstrahlung spectrum. As an example, I have simulated the ROSAT counting rates for Nova GQ Mus spectra as deduced from EXOSAT countrates. Figure 3 shows the differential countrates per energy interval as a function of the detector energy for the two spectral models of blackbody and thermal bremsstrahlung, after smearing the energy with the ROSAT PSPC resolution. The total countrate expected in ROSAT for both cases is about $0.015 \mathrm{cts} \mathrm{s}^{-1}$, implying that in an exposure of $10^{4} \mathrm{~s}$ we should get around 150 source counts. It is evident from the figure that we should have no difficulty in distinguishing the two spectra.

One main scientific objective of ROSAT is to do an all-sky survey in a scanning mode during the first six months of the mission. During this period most of the sky will be covered at a typical exposure of $10^{3} \mathrm{~s}$ with typical detection sensitivities around $0.015 \mathrm{cts} \mathrm{s}^{-1}$ (that which is expected from Nova GQ Mus like source). This implies that during the survey we should be able to detect most galactic novae brighter than 8 magnitudes that have gone off in the 3-4 year interval prior to the survey. However, the total counts collected per nova would be too meager to do any decent spectral investigation. A fortunate aspect of the ROSAT all-sky survey is that the Large Magellanic Cloud (LMC) will be observed for about $5000 \mathrm{~s}$, owing to its high ecliptic lattitude position. Although novae in LMC are on the average a factor 10 more distant than the galactic novae, due to the low column density to LMC $\left(\sim 9 \times 10^{20} \mathrm{~cm}^{-2}\right)$, for the hot white dwarf model we expect the same countrate for a Nova GQ Mus like source in LMC as the counrate expected if it was in its normal position in our galaxy, i.e. $0.015 \mathrm{cts} \mathrm{s}^{-1}$. Consequently, the ROSAT survey should detect several novae from LMC if the hot white dwarf model is correct. Due to the $\sim 0.5^{\prime}$ angular resolution of the survey we may however, suffer from source confusion problems. In the pointed observations phase following the all-sky survey ROSAT should be able to detect a number 


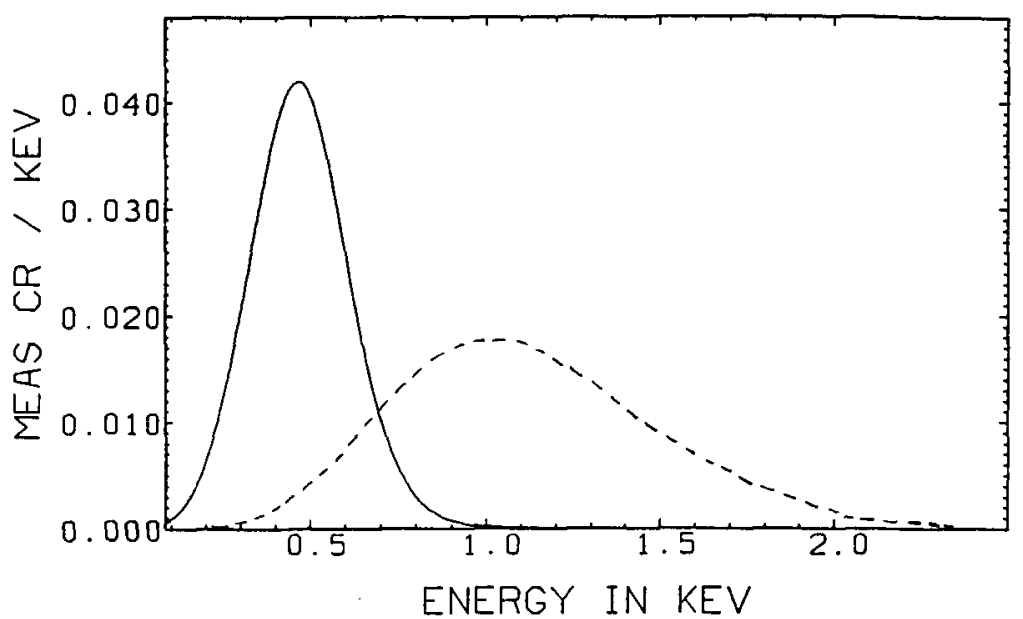

Figure 3: The simulated ROSAT differential counrate per energy interval as a function of the detector energy for Nova GQ Mus after smearing the detected counts with the PSPC energy resolution. The input flux parameters are as determined from the EXOSAT observations. The solid curve is that which would be expected from a hot white dwarf remnant radiating $5 \times 10^{36}$ erg $\mathrm{s}^{-1}$ at an effective blackbody temperature of $400000 \mathrm{~K}$. The dashed curve is that which would be expected from a shocked gas radiating $4 \times 10^{34} \mathrm{erg} \mathrm{s}^{-1}$ at a temperature of $2 \mathrm{keV}$. The total countrates are about $0.015 \mathrm{cts} \mathrm{s}^{-1}$ in both cases.

of recent novae. These X-ray observations should contribute significantly to our understanding of the novae phenomena.

\section{REFERENCES}

Brecher, K., Ingham, W.H., and Morrison, P.: 1977, Astrophys. J., 213, 492

Cruise, A.M.: 1977, Nature, 267, 685

Chlebowski, T. and Kałużny, J.: 1988, Acta Astron., 38, 329

Gallagher, J.S., and Starrfield, S.: 1978, Ann. Rev. Astr. Ap. , 16, 121

Hoffman, J.A., Lewin, W.H.G., Brecher, K., Buff, J., Clark, G.W., Joss, P.C., and Matilsky, T.: 1976, Nature, 261, 208

Hutchings, J.B.: 1980, Pub. A.S.P. , 92, 458

Krautter, J. and Williams, R.E.: 1989, Ap. J., in press

MacDonald, J.: 1980, Mon. Not. R. astr. Soc., 191, 933

Mason, K.O., Cordova, F.A., Bode, M.F., and Barr, P.: 1986, in RS Oph and the Recurrent Novae Phenomenon, ed M.F. Bode (VNU press; Utrecht), p. 167

Ögelman, H., Beuermann, K. and Krautter, J.: 1984, Astrophys. J. (Letters), 287, L31

Ögelman, H., Krautter, J. and Beuermann, K.: 1987, Astron. Astrophys., 177, 110

Paczyński, B.: 1971, Acta Astron. , 21, 417 
Starrfield, S.: 1988, in Multiwavelength Astrophysics, ed. F. Cordova (Cambridge: University Press), p. 159

Starrfield, S., Truran, J.W., Sparks, W.M. and Krautter, J.: 1989, to appear in the proceeding of the Berkeley UV Conference.

Trümper, J.: 1983, Adv. Space Res., 2, 241

Truran, J.W.: 1982, in Essays in Nuclear Astrophysics, ed. C.A. Barnes, D.D. Clayton, and D.N. Schramm (Cambridge: Cambridge University Press), p. 467 